\title{
French American British (FAB) morphological classification of childhood lymphoblastic leukaemia and its clinical importance
}

\author{
J S LILLEYMAN,* I M HANN, $\dagger$ R F STEVENS, $\ddagger$ O B EDEN, $\S$ S M RICHARDS \\ On behalf of the Medical Research Council's Working Party on Leukaemia in Childhood \\ From the *Children's Hospital, Sheffield, the †Royal Hospital for Sick Children, Glasgow, the $\ddagger$ Royal \\ Manchester Children's Hospital, Manchester, the §Royal Hospital for Sick Children, Edinburgh, and the \\ TClinical Trials Service Unit, Oxford
}

SUMMARY As part of the Medical Research Council Leukaemia Trial UKALL VIII, 738 unselected children with acute lymphoblastic leukaemia (ALL) had the morphology of their marrow blast cells $₫$ reviewed by a panel of three haematologists. Ninety four $(13 \%)$ showed appearances classifiable as 윽 type $\mathrm{L}_{2}$ by the French American and British (FAB) cooperative group's criteria, five $(0.7 \%)$ were typed $L_{3}$, and the remaining $639(86 \%)$ as $L_{1}$.

Disregarding the patients classified as $L_{3}$, those with the $L_{2}$ variant showed an inferior disease $\frac{\mathbb{D}}{\mathbb{D}}$ free survival to that of the remainder $(\mathrm{p}<0.01)$, and more of them failed to remit after receiving 3 "standard" remission induction treatment $(p<0.01)$. They included an excess of older children $\stackrel{\Phi}{工}$ ( $p<0.01$ ) with less profound marrow failure at diagnosis, and fewer of them expressed the common $\vec{\bullet}$ ALL antigen $(p=0.05)$. There was no association between $L_{2}$ morphology and the diagnostic whige cell count, sex, or the presence of a mediastinal mass.

These findings confirm earlier reports that FAB $\mathrm{L}_{2}$ ALL is associated with a poor prognosis argi that it occurs more commonly in older children. The high remission failure rate is a recent observation and indicates that alternative early treatment may be appropriate for such patients.

Since effective treatment for childhood lymphoblastic leukaemia (ALL) was developed repeated attempts have been made to identify clinical and pathological features related to prognosis. These efforts have been directed at refining treatment protocols and helping with the classification of what is clearly a heterogeneous group of disorders.

Blast cell morphology has recently enjoyed a renaissance as a prognostic factor, chiefly due to the introduction of the French American and British (FAB) cooperative group's classification of acute lymphoblastic leukaemia (ALL). ${ }^{12}$ Using their criteria, several authors in the past few years have suggested that the minority $L_{2}$ variant is associated with a worse outlook..$^{3-8}$ None the less, several unanswered questions remain. Can $\mathrm{L}_{2}$ disease be reproducibly identified by independent morphologists? What is its prevalence in a large

Accepted for publication 23 April 1986 unselected series? Is the association with poor treat- $\overrightarrow{\overrightarrow{0}}$ ment response due to a higher relapse rate or lower 3 remission induction rate? Is $\mathbf{L}_{2}$ morphology of independent prognostic importance or is it associated with some other well known adverse feature such as older age, higher presenting leucocyte count, or male sex? In an attempt to answer some or all of these 3 questions our study was conducted as part of a large multicentre trial.

Patient and methods

All children who were entered into the Medical

Members of the Working Party:

Professor RM Hardisty (Chairman); Dr CC Bailey; Dr P Barbor; $\stackrel{0}{0}$ Dr ND Barnes; Dr C Barton; Dr SC Cartwright; Dr AW Craft; Dr JM Chessells; Dr SI Dempsey; Dr JH Durrant; Dr OB Eden; $\sigma$ Dr P Emerson; Professor JJ Fennelly; Professor DAG Galton; Dr IM Hann; Dr FGH Hill; Dr J Kernaham; Dr JS Lilleyman; Professor TJ McElwain; Dr JR Mann; Dr J Martin; Dr PH Morris-Jones; Dr A Oakhill; Professor J Peto; Dr M Radford; $\stackrel{\mathscr{C}}{+}$ Dr JKH Rees; Dr S Richards; Dr RF Stevens; Dr GP Summerfield; $\square$ Dr EN Thompson. 
Table $1{ }^{*} F A B$ type scoring: $L_{1} v L_{2}$

\begin{tabular}{lc}
\hline Feature & Score \\
\hline$* *$ Nuclear: cytoplasmic ratio high in $>75 \%$ cells & +1 \\
Nuclear: cytoplasmic ratio low in $>25 \%$ cells & -1 \\
Nucleoli absent or inconspicuous in $>75 \%$ cells & +1 \\
Nucleoli present in $>25 \%$ cells & -1 \\
Irregular nuclear membrane in $<25 \%$ cells & 0 \\
Irregular nuclear membrane in $>25 \%$ cells & -1 \\
\$Large cells $<50 \%$ of total & 0 \\
Large cells $>50 \%$ of total & -1 \\
Totals possible & +2 to -4 \\
\hline
\end{tabular}

*FAB type $L_{1}=0,1,+2 ; L_{2}=-1,-2,-3,-4 ;{ }^{* *}$ cytoplasm occupies $<20 \%$ of cell surface area; $\$$ "large" $=$ more than $2 \times$ diameter of small lymphocyte.

Research Council Childhood Leukaemia Trial UKALL VIII had diagnostic bone marrow smears submitted for central review by a panel of three haematologists. Slides were mostly stained in the review laboratory but some were stained elsewhere. As a result, various Romanowsky staining techniques were used, May-Grünwald-Giemsa being the most common.

Inadequate slides were rejected. They were regarded as inadequate if they were badly spread (usually too thickly) or not spread at all but squashed. Dilute aspirates yielding a preparation with less than two cells per high power field were also rejected. If no satisfactory slides were available then that patient was excluded from the study.

Each morphologist reviewed each slide blind, knowing only the name of the patient but no clinical details. The study was carried out prospectively, and there was no possibility of knowing the clinical outcome of any patient's disease.

Each panel member scored each marrow slide $L_{1}$ or $\mathrm{L}_{2}$ strictly according to the FAB group's criteria (table 1). Indeterminate or intermediate criteria scored neither positively nor negatively. Each cell feature was expressed by each member as a percentage, and where the difference between observers for a given feature was $<10 \%$, the final FAB score for that feature was calculated as a mean of the three observers' estimates. Where a greater discrepancy arose (usually where slide quality was borderline), the slide was either sent for arbitration to two members of the original FAB group, or, towards the end of the study, was typed on the basis of the majority view-that is, two of the three panellists. The third FAB type, $L_{3}$ disease, was generally easily recognised and not scored in the same way.

The UKALL VIII study and trial were open to all children under 14 years old with any type of ALL. The treatment schedule has been published elsewhere. ${ }^{9}$ Disease free survival was estimated in the usual way, and all statistical analyses were carried out at the Clinical Trials Service Unit at the Radcliffe
Infirmary, Oxford. Median follow up time for this analysis was three years.

\section{Results}

Of 833 patients entered into UKALL VIII between September 1981 and December 1984, 95 (11\%) either had no slides submitted for FAB typing or had their slides discarded as unsuitable. Of the remaining 738 , $639(86 \%)$ were typed as $L_{1}, 94(13 \%)$ as $L_{2}$, and five $(0 \cdot 7 \%)$ as the rare but easily identified $L_{3}$.

\section{CONCORDANCE BETWEEN OBSER VERS}

Where slides were not eventually rejected as inadequate, the panel failed to agree on the FAB type in 63 patients (8.5\%). None the less all 63 were typed; in 25 the arbitrating view of two members of the FAB group was sought, and in the remainder the majority type (two of the three opinions) was taken as correct.

\section{DISEASE FREE SURVIVAL}

Fig 1 shows disease free survival for all 738 patients classified by their FAB type. The patients with $L_{2}$ disease show a significantly inferior prognosis to that of the group with $L_{1}$ disease $(p=0.01)$, and the group with $L_{3}$ disease fared worse still.

Fig 2 shows disease free survival for the 730 patients who scored plus or minus for the presence of nucleoli, and fig 3 that for the 716 who similarly scored plus or minus (as opposed to no score if equivocal) for a low $v$ a high nuclear:cytoplasmic ratio. Both of these features individually achieved conventional significance as unstratified prognostic variables, but if one is stratified by the other the significance is lost, suggesting that cells tend to have both features or neither.

Nuclear membrane irregularity in $>25 \%$ of cells

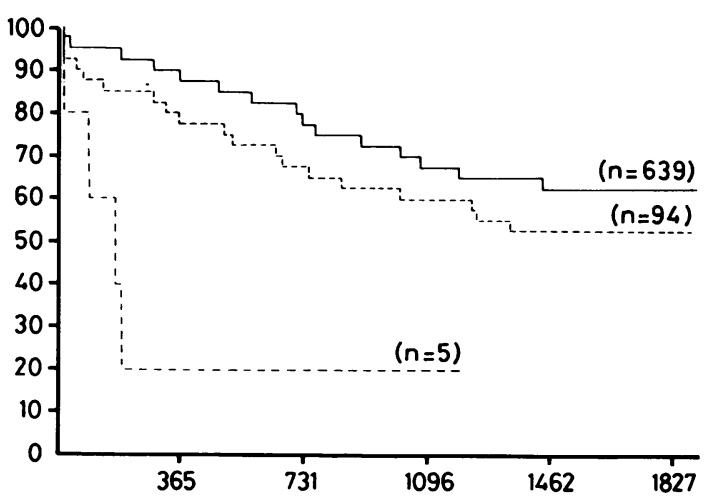

Fig 1 UKALL VIII January 1986: actuarial disease free survival indicated by $F A B$ type. Time scale in days: $L_{1}=(n=639) ; L_{2}=(n=94) ; L_{3}=(n=5)$. Difference between $L_{1}$ and $L_{2}: p=0.01$ (logrank). 


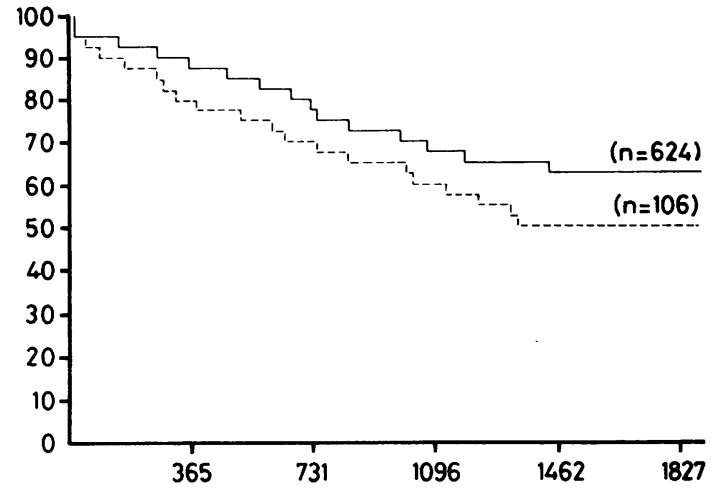

Fig 2 UKALL VIII January 1986: actuarial disease free survival indicated by presence or absence of nucleoli in $>25 \%$ blast cells. Time scale in days: $<25 \%=(n=624)$; $>25 \%=(n=106)$.

(seen in $78(11 \%)$ of patients) and large cell size (seen in $49(7 \%)$ were not significantly associated with prognosis, though there was a trend for both features to be associated with a higher relapse rate. Table 2 shows the numbers of patients assessed for each FAB feature and the proportion relapsing or dying, or both, in each category.

ASSOCIATION WITH OTHER CLINICAL FEATURES FAB type was not associated with sex or diagnostic white cell count but was associated with age (table 3 ). Even after adjusting for age, $\mathrm{L}_{2}$ disease was still associated with a poorer disease free survival $(p=0.03)$. The trend for an increasing proportion of patients over the age of 10 years to have $L_{2}$ disease may have been partly balanced by an excess of patients with $L_{2}$ disease in the small proportion of children under a year old (four of $17(23 \%)$ ).

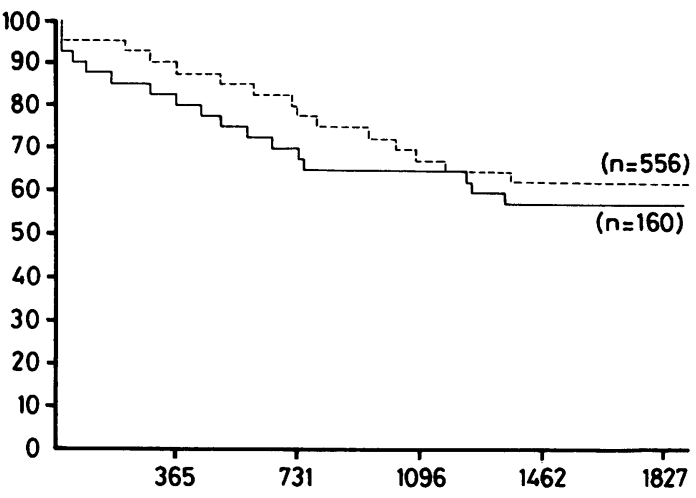

Fig 3 UKALL VIII January 1986: actuarial disease free survival indicated by presence of low nuclear: cytoplasmic ratio in $>$ or $<25 \%$ blast cells. Time scale in days. $<25 \%=(n=556) ;>25 \%=(n=160)$.

As for other clinical features, there was a significant trend to higher presenting haemoglobin values $(p=0.05)$ and platelet counts $(p>0.02)$ in the group with $\mathrm{L}_{2}$ disease, and their cells showed a lower incidence of expression of the common acute lymphoblastic leukaemia (CALL) antigen $(p=0.05)$.

\section{FAB TYPE AND PATTERN OF ADVERSE EVENTS}

Patients with $\mathrm{L}_{2}$ disease did not develop central ner 8 vous system or testicular disease more often, but the did develop bone marrow recurrence more often Significantly more of them failed to remit $(p<0.001 ?$ table 4), and there was a suggestion that they had shorter subsequent survival if they relapsed after achieving remission $(p=0.07)$.

NON-TYPED PATIENTS

Ninety five $(11 \%$ of the total) patients had no FAB

Table 2 UKALL VIII: deaths and relapses in morphologically defined sub groups

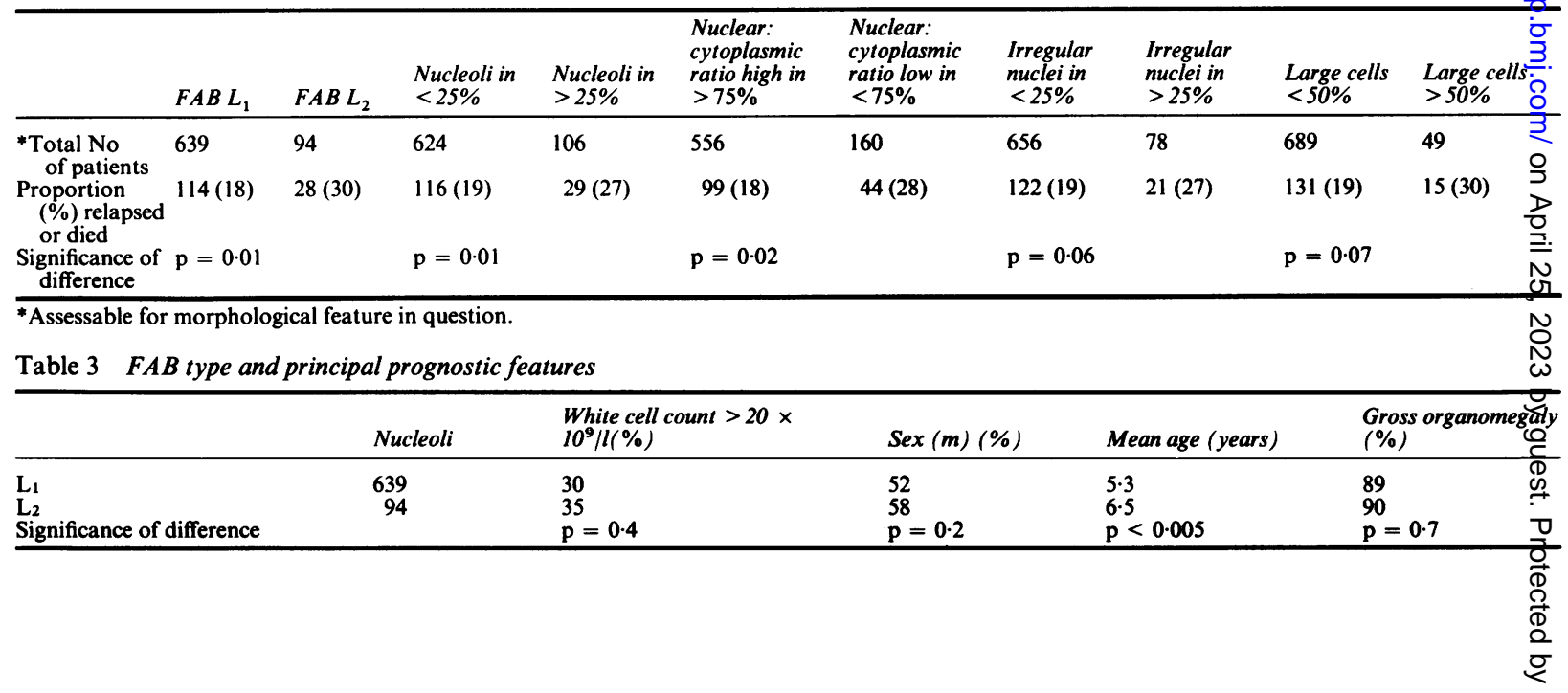


Table 4 FAB type and adverse events (figures in parentheses are numbers \%)

\begin{tabular}{lrrrl}
\hline & No & $L_{1}(\%)$ & $L_{2}(\%)$ & Significance of difference \\
\hline Total & 733 & $639(87)$ & $94(13)$ & \\
Relapsed or died & 215 & $178(83)$ & $37(17)$ & $\mathrm{p}=0.01^{*}$ \\
Failed to enter remission & 32 & $23(72)$ & $9(28)$ & $\mathrm{p}<0.02 \S$ \\
Failed to enter remission despite & 15 & $8(53)$ & $7(47)$ & $\mathrm{p}<0.001 \S$ \\
$\quad$ surviving $>28$ days & & & & \\
\hline
\end{tabular}

*Logrank.

$\S \chi^{2}$ with Yates' correction.

type recorded, either because no slides were received for review, or because the material submitted was technically inadequate. At the time of this report data on the fate of 91 were available: $33(36 \%)$ had relapsed or died, which is a slightly higher proportion than that for the FAB typed group (219 of 738 or $30 \%$ ). It may be that the group of 95 contained an excess of some subgroup, perhaps those with severe secondary myelofibrosis, which would have made marrow aspiration difficult. They seemed to be randomly distributed between the sexes and different age groups.

\section{Discussion}

The three morphological groups of ALL defined by the FAB group ${ }^{12}$ are grossly unevenly distributed. We found a prevalence of $86 \%, 13 \%$, and $1 \%$ for $L_{1}$, $L_{2}$, and $L_{3}$ types, respectively, in a group of 738 consecutive patients.

Dismissing the tiny group with $\mathbf{L}_{\mathbf{3}}$ disease as morphologically, immunologically, cytogenetically, and clinically distinct-they are the $B$ cell leukaemias - the main interest in typing the other ALLs by light microscopy was twofold. First, to find out whether they could be objectively and reproducibly subcategorised into two groups, and secondly, if so, whether such a classification had any clinical relevance.

Reliable typing does seem possible. Using the FAB group's scoring system, ${ }^{2}$ the measure of agreement reached between three independent haematologists in this study was as good as could reasonably be expected, the slides over which there was strong disagreement were usually of borderline technical adequacy. It was usually obvious whether a given case should be typed as $L_{1}$ or $L_{2}$, even if the estimated proportion of cells showing a particular feature differed between observers.

Some conclusions can be drawn from the UKALL VIII patients about the relevance of such a classification. $\mathrm{L}_{2}$ ALL occurs more commonly in older children, is associated with less profound bone marrow failure, and it also more often does not express the common ALL antigen. Apart from these distinguishing clinical features, there is also a trend emerging for patients with $\mathrm{L}_{2}$ disease to have a worse prognosis. Furthermore, the inferior disease free survival they display is not related to age, sex, or presenting white cell count. It may, in part, be due to the relatively high proportion of them who fail to achieve remission. For those who do remit there is a higher marrow relapse rate but no excess of extramedullary disease. Survival after relapse may be shorter for patients with $L_{2}$ disease than for those with $L_{1}$ disease.

Some of these observations are new; others are not. The worse prognosis for patients with $\mathrm{L}_{2}$ disease was first noted in 1978 by a Hungarian group ${ }^{3}$ and later confirmed by Hann et al in the United Kingdom. ${ }^{4}$ Viana $e t a l^{5}$ not only indicated that patients with $\mathrm{L}_{2}$ disease had a poorer outlook but noted that more of them were older. This association with age was also commented on by the FAB group in 1981 when they refined their classification to include a scoring system for $L_{1}$ and $L_{2}{ }^{2}$ Revision of their original criteria ${ }^{1}$ meant that fewer children were classified as $L_{2}(16 \%$ in their index series), but they did note that the prevalence of $\mathrm{L}_{2}$ disease was positively correlated with age.

The United States Children's Cancer Study Group (CCSG) modified the FAB scoring system further; and they assessed each case on a cell by cell basisthat is, calling each cell $\mathbf{L}_{1}$ or $\mathbf{L}_{2}$ - with each patient being classified according to the proportion of $L_{2}$ cells present. ${ }^{67}$ This differs from the FAB scoring system, which is done on a feature by feature basis - that is, the proportion of cells showing a low nuclear: cytoplasmic ratio and so on-and where a final type is ascribed on the basis of a composite score from each of four features: ${ }^{2}$ Such a superficially subtle difference may be quite important and might explain why the CCSG recently found FAB type to be the second most important independent prognostic factor, which indicates speed of therapeutic response. ${ }^{7}$ It might also explain why fewer of their patients $(<10 \%)$ type as L2.

The CCSG also noted in their " 160 series" that the 37 patients with "pure" $L_{2}$ disease (those with $>50 \%$ $\mathrm{L}_{2}$ cells) had a $16 \cdot 3 \%$ remission failure rate compared with $3.5 \%$ for the 873 children with $L_{1}$ disease. The implicit suggestion contained in these figures is supported by our findings. Few patients with ALL fail to remit on current regimens, and there were only 15 such children in the whole of UKALL VIII, if failing 
to remit is defined as having detectable disease after 28 days of treatment. Seven of them typed as $\mathrm{L}_{2} ;$ a highly significant excess. Other new findings arising from our analysis are that $L_{2} A L L$ is associated with less severe marrow failure at presentation and that it less commonly expresses the "common" ALL antigen; but these findings need to be confirmed.

The clinical importance of ALL FAB type seems to be fairly clear. $\mathrm{L}_{2}$ ALL is a more refractory disease and so is a logical candidate for alternative treatment. Such treatment should be different from the beginning, in view of the high remission failure rate. Whether $\mathbf{L}_{2}$ disease is a nosologically distinct disorder is less certain. If it is the CCSG may have defined it more precisely with their exclusive classification. Further information might be obtained from parallel cytogenetic studies, or from a similar morphological analysis of a large group of adults with ALL.

\section{References}

1 Bennett JM, Catovsky D, Daniel MT, et al. Proposals for the classification of the acute leukaemias. $\mathrm{Br} J$ Haematol 1976;33:451-8.

2 Bennett JM, Catovsky D, Daniel MT, et al. The morphological classification of acute lymphoblastic leukaemia: concordance among observers and clinical correlations. $\mathrm{Br} \mathrm{J}$ Haematol 1981;47:553-61.
3 Keleti J, Revesz T, Schuler D. Morphological diagnosis in childhood leukaemia. $\mathrm{Br} J$ Haematol 1978;40:501-6.

4 Hann IM, Evans DIK, Palmer MK, Morris Jones P, Haworth C. The prognostic significance of morphological features in childhood acute lymphoblastic leukaemia. Clin Lab Haematol 1979;1:215-26.

5 Viana MB, Maurer HS, Ferenc C. Subclassification of acute lymphoblastic leukaemia in children: analysis of the reproducibility of morphological criteria and prognostic implications. Br J Haematol 1980;44:383-8.

6 Miller DR, Leikin S, Albo V, Sather H, Hammond D. Prognostic importance of morphology (FAB Classification) in childhood acute lymphoblastic leukaemia. $\mathrm{Br} J$ Haematol 1981;48: 199-206.

7 Miller DR, Krailo M, Bleyer WA, et al. Prognostic implications of blast cell morphology in childhood acute lymphoblastic leukemia: a report from the Children's Cancer Study Group. Cancer Treatment Rep 1985;69:1211-21.

8 Kalwinsky DK, Roberson P, Dahl G, et al. Clinical relevance of lymphoblast biological features in children with acute lymphoblastic leukaemia. J Clin Oncol 1985;3:477-89.

9 Report to the Council by the Working Party on Leukaemia in Childhood. Improvement in treatment for children with acute lymphoblastic leukaemia. The Medical Research Council UKALL Trials 1972-84. Lancet 1986;i:408-11.

Requests for reprints to: Dr JS Lilleyman, Department of Haematology, The Children's Hospital, Western Bank, Sheffield S10 2TH, England. 